\title{
A weak Kellogg property for quasiminimizers
}

\author{
Anders Björn
}

\begin{abstract}
The Kellogg property says that the set of irregular boundary points has capacity zero, i.e. given a bounded open set $\Omega$ there is a set $E \subset \partial \Omega$ with capacity zero such that for all $p$-harmonic functions $u$ in $\Omega$ with continuous boundary values in Sobolev sense, $u$ attains its boundary values at all boundary points in $\partial \Omega \backslash E$.

In this paper, we show a weak Kellogg property for quasiminimizers: a quasiminimizer with continuous boundary values in Sobolev sense takes its boundary values at quasievery boundary point. The exceptional set may however depend on the quasiminimizer. To obtain this result we use the potential theory of quasisuperminimizers and prove a weak Kellogg property for quasisuperminimizers.
\end{abstract}

This is done in complete doubling metric spaces supporting a Poincaré inequality.

Mathematics Subject Classification (2000). Primary 31C45; Secondary 35J65, 49J27, $49 \mathrm{~N} 60$.

Keywords. Doubling measure, weak Kellogg property, metric space, nonlinear, $p$-harmonic, Poincaré inequality, potential theory, quasiharmonic, quasiminimizer, quasisuperharmonic, quasisuperminimizer, regular.

\section{Introduction}

Let $\Omega \subset \mathbb{R}^{n}$ be a bounded open set, $Q \geq 1$ and $1<p<\infty$, then a function $u$ in the Sobolev space $W_{\text {loc }}^{1, p}(\Omega)$ is a $Q$-quasiminimizer (of the $p$-Dirichlet integral $\left.\int|\nabla u|^{p} d x\right)$ in $\Omega$ if

$$
\int_{\varphi \neq 0}|\nabla u|^{p} d x \leq Q \int_{\varphi \neq 0}|\nabla(u+\varphi)|^{p} d x
$$

for all functions $\varphi \in W^{1, p}(\Omega)$ with $\operatorname{supp} \varphi \Subset \Omega$. A 1-quasiminimizer, usually called a minimizer, is a weak solution of the corresponding Euler equation

$$
\operatorname{div}\left(|\nabla u|^{p-2} \nabla u\right)=0 .
$$


Quasiminimizers were introduced by Giaquinta-Giusti [13], [14]. Quasiminimizers have a rigidity that weak solutions lack: if a variational kernel $F(x, \nabla u)$ satisfies

$$
a|h|^{p} \leq F(x, h) \leq b|h|^{p} \text { for some } 0<a \leq b<\infty,
$$

then the quasiminimizers of $\int F(x, \nabla u) d x$ coincide with those of $\int|\nabla u|^{p} d x$ (although the constant $Q$ may change).

Giaquinta-Giusti [14] proved several fundamental properties for quasiminimizers including the interior regularity result that a quasiminimizer can be modified on a set of zero measure so that it becomes Hölder continuous. These results were extended to metric spaces by Kinnunen-Shanmugalingam [24].

For $f \in C(\partial \Omega)$, the Perron method provides a unique solution $u$ of the Dirichlet problem (the boundary value problem), i.e. $u$ is $p$-harmonic in $\Omega$ and takes the boundary values $f$ in Sobolev sense. A point $x_{0} \in \partial \Omega$ is said to be regular if $\lim _{\Omega \ni y \rightarrow x_{0}} u(y)=f\left(x_{0}\right)$ for every $f \in C(\partial \Omega)$. (A p-harmonic function is a continuous minimizer and a quasiharmonic function is a continuous quasiminimizer. For the relation between Sobolev and Perron solutions of the Dirichlet problem, see Björn-Björn-Shanmugalingam [8] and Björn-Björn [6].)

The Kellogg property says that the set of irregular boundary points has zero capacity. For $n=p=2$ the Kellogg property was proved by Kellogg [18] in 1928, and for $n \geq 3$ and $p=2$ it was proved by Evans [12] in 1933. For $1<p<\infty$ the Kellogg property was obtained for weighted Euclidean spaces in Heinonen-KilpeläinenMartio [15], Theorem 9.11. See [15], p. 192, for more on the history in the nonlinear case.

More recently, the theory of $p$-harmonic functions has been extended to complete metric spaces equipped with a doubling measure supporting a Poincaré inequality. In this generality the Kellogg property was proved in Björn-Björn-Shanmugalingam [7], Theorem 3.9.

Boundary regularity for quasiminimizers has been studied by Ziemer [28] in $\mathbb{R}^{n}$ and J. Björn [10] in metric spaces, they both obtained sufficient conditions for a boundary point to be regular for quasiharmonic functions. Recently, J. Björn [11] showed that open sets can be approximated by sets that are regular for quasiharmonic functions, see Theorem 5.3.

The main result of this paper is a weak Kellogg property for quasiharmonic functions, Theorem 4.1. Here the exceptional set of irregular boundary points depends on the quasiharmonic function, but has capacity zero. It should be observed that when the Kellogg property is used in various applications often just one function is involved and what is really needed is the weak Kellogg property.

There is no uniqueness in the Dirichlet problem for quasiminimizers, nor any comparison principle. Also, being a $Q$-quasiminimizer is not a local property. This leads to difficulties not present in the theory of minimizers. In order to obtain the weak Kellogg property for quasiharmonic functions we use the potential theory of quasimin- 
imizers, which includes quasisuperminimizers and quasisuperharmonic functions, recently developed by Kinnunen-Martio [23].

In this paper we also obtain a weak Kellogg property for quasisuperharmonic functions, Theorem 4.4, and give a list of characterizations of quasiminimizers and quasisuperminimizers, some of which seem to be new.

For more on quasiminimizers and their importance see the introduction in Kinnunen-Martio [23]. An application of the weak Kellogg property for quasiharmonic functions will be given in the forthcoming paper A. Björn [4]. For removable singularities for quasiharmonic and quasisuperharmonic functions, see A. Björn [3].

For examples of complete metric spaces equipped with a doubling measure supporting a Poincaré inequality, see, e.g., A. Björn [1], [2].

Acknowledement. The author was supported by the Swedish Research Council and Gustaf Sigurd Magnuson's fund of the Royal Swedish Academy of Sciences. These results were partially obtained while the author was visiting the Department of Mathematical Analysis at the Charles University in Prague during the autumn 2003.

\section{Notation and preliminaries}

We assume throughout the paper that $X=(X, d, \mu)$ is a complete metric space endowed with a metric $d$ and a doubling measure $\mu$, i.e. there exists a constant $C>0$ such that for all balls $B=B\left(x_{0}, r\right):=\left\{x \in X: d\left(x, x_{0}\right)<r\right\}$ in $X$ (we make the convention that balls are nonempty and open),

$$
0<\mu(2 B) \leq C \mu(B)<\infty,
$$

where $\lambda B=B\left(x_{0}, \lambda r\right)$. We emphasize that the $\sigma$-algebra on which $\mu$ is defined is obtained by the completion of the Borel $\sigma$-algebra. We also assume that $1<p<\infty$. (At the end of this section we make some further assumptions assumed in the rest of the paper.)

Note that some authors assume that $X$ is proper rather than complete, but, since $\mu$ is doubling, $X$ is proper if and only if it is complete.

Definition 2.1. A nonnegative Borel function $g$ on $X$ is an upper gradient of an extended real-valued function $f$ on $X$ if for all nonconstant rectifiable paths $\gamma:\left[0, l_{\gamma}\right] \rightarrow X$

$$
\left|f(\gamma(0))-f\left(\gamma\left(l_{\gamma}\right)\right)\right| \leq \int_{\gamma} g d s
$$

whenever both $f(\gamma(0))$ and $f\left(\gamma\left(l_{\gamma}\right)\right)$ are finite, and $\int_{\gamma} g d s=\infty$ otherwise. If $g$ is a nonnegative measurable function on $X$ and if (2.1) holds for $p$-almost every path, then $g$ is a $p$-weak upper gradient of $f$. 
By saying that (2.1) holds for $p$-almost every path we mean that it fails only for a path family with zero $p$-modulus, see Definition 2.1 in Shanmugalingam [26]. It is implicitly assumed that $\int_{\gamma} g d s$ is defined (with a value in $[0, \infty]$ ) for $p$-almost every rectifiable path.

If $g \in L^{p}(X)$ is a $p$-weak upper gradient of $f$, then one can find a sequence $\left\{g_{j}\right\}_{j=1}^{\infty}$ of upper gradients of $f$ such that $g_{j} \rightarrow g$ in $L^{p}(X)$, see Lemma 2.4 in Koskela-MacManus [25].

If $f$ has an upper gradient in $L^{p}(X)$, then it has a minimal $p$-weak upper gradient $g_{f} \in L^{p}(X)$ in the sense that $g_{f} \leq g \mu$-a.e. for every $p$-weak upper gradient $g \in L^{p}(X)$ of $f$, see Corollary 3.7 in Shanmugalingam [27].

If $f, h \in N^{1, p}(X)$, then $g_{f}=g_{h} \mu$-a.e. in $\{x \in X: f(x)=h(x)\}$, in particular $g_{\min \{f, c\}}=g_{f} \chi_{f \neq c}$ for $c \in \mathbb{R}$. For these and other facts on $p$-weak upper gradients, see, e.g., Björn-Björn [5], Section 3.

Definition 2.2. We say that $X$ supports a weak $(1, q)$-Poincaré inequality if there exist constants $C>0$ and $\lambda \geq 1$ such that for all balls $B \subset X$, all measurable functions $f$ on $X$ and all upper gradients $g$ of $f$,

$$
f_{B}\left|f-f_{B}\right| d \mu \leq C(\operatorname{diam} B)\left(f_{\lambda B} g^{q} d \mu\right)^{1 / q},
$$

where $f_{B}:=f_{B} f d \mu:=\int_{B} f d \mu / \mu(B)$.

In the definition of Poincaré inequality we can equivalently assume that $g$ is a $q$-weak upper gradient - see the comments above. It is also equivalent to require that (2.2) holds for all $f \in \operatorname{Lip}_{c}(X)$ and all upper gradients $g \in \operatorname{Lip}_{c}(X)$ of $f$, see Keith [16], Theorem 2. We say that a set $E \Subset A$ if $\bar{E}$ is a compact subset of $A$, and let $\operatorname{Lip}_{c}(A)=\{f \in \operatorname{Lip}(A): \operatorname{supp} f \Subset A\}$.

Following Shanmugalingam [26], we define a version of Sobolev spaces on the metric space $X$.

Definition 2.3. Whenever $u \in L^{p}(X)$, let

$$
\|u\|_{N^{1, p}(X)}=\left(\int_{X}|u|^{p} d \mu+\inf _{g} \int_{X} g^{p} d \mu\right)^{1 / p},
$$

where the infimum is taken over all upper gradients of $u$. The Newtonian space on $X$ is the quotient space

$$
N^{1, p}(X)=\left\{u:\|u\|_{N^{1, p}(X)}<\infty\right\} / \sim,
$$

where $u \sim v$ if and only if $\|u-v\|_{N^{1, p}(X)}=0$. 
The space $N^{1, p}(X)$ is a Banach space and a lattice, see Shanmugalingam [26].

Definition 2.4. The capacity of a set $E \subset X$ is the number

$$
C_{p}(E)=\inf \|u\|_{N^{1, p}(X)}^{p},
$$

where the infimum is taken over all $u \in N^{1, p}(X)$ such that $u=1$ on $E$.

The capacity is countably subadditive. For this and other properties as well as equivalent definitions of the capacity we refer to Kilpeläinen-Kinnunen-Martio [19] and Kinnunen-Martio [20], [21].

We say that a property regarding points in $X$ holds quasieverywhere (q.e.) if the set of points for which the property does not hold has capacity zero. The capacity is the correct gauge for distinguishing between two Newtonian functions. If $u \in N^{1, p}(X)$, then $u \sim v$ if and only if $u=v$ q.e. Moreover, Corollary 3.3 in Shanmugalingam [26] shows that if $u, v \in N^{1, p}(X)$ and $u=v \mu$-a.e., then $u \sim v$.

Further, if $X$ supports a weak $(1, p)$-Poincaré inequality and $\mu$ is doubling, then Lipschitz functions are dense in $N^{1, p}(X)$ and the functions in $N^{1, p}(X)$ are quasicontinuous, see [26] and Björn-Björn-Shanmugalingam [9]. This means that in the Euclidean setting, $N^{1, p}\left(\mathbb{R}^{n}\right)$ is the refined Sobolev space as defined on p. 96 of Heinonen-Kilpeläinen-Martio [15]. It also follows that if $X$ supports a weak $(1, p)$-Poincaré inequality and $\Omega \subset X$ is open, then the functions in $N^{1, p}(\Omega)$ are all quasicontinuous, see, e.g., [9].

To be able to compare the boundary values of Newtonian functions we need a Newtonian space with zero boundary values. Let $\Omega$ be an arbitrary nonempty open subset of $X$, and let

$$
N_{0}^{1, p}(\Omega)=\left\{\left.f\right|_{\Omega}: f \in N^{1, p}(X) \text { and } f=0 \text { on } X \backslash \Omega\right\} .
$$

One can replace the assumption " $f=0$ on $X \backslash \Omega$ " with " $f=0$ q.e. on $X \backslash \Omega$ " without changing the obtained space $N_{0}^{1, p}(\Omega)$. Note that if $C_{p}(X \backslash \Omega)=0$, then $N_{0}^{1, p}(\Omega)=N^{1, p}(\Omega)$. Note also that

$$
N_{0}^{1, p}(\Omega)=\left\{\left.f\right|_{\Omega}: f \in N^{1, p}(\bar{\Omega}) \text { and } f=0 \text { on } \partial \Omega\right\} .
$$

Here we have used the nonstandard Sobolev space $N^{1, p}(\bar{\Omega})$ on a closed subset. Of course, $\bar{\Omega}$ is a complete metric space although $\left.\mu\right|_{\bar{\Omega}}$ may not be doubling. Defining the Newtonian space is however not depending on this assumption. For a reader mainly just interested in $\mathbb{R}^{n}$ the space $N^{1, p}(\bar{\Omega})$ appearing in several of the theorems in this paper may be puzzling. However it is trivial that $N^{1, p}(X) \subset N^{1, p}(\bar{\Omega})$ and not much is lost if the reader replaces $N^{1, p}(\bar{\Omega})$ by $N^{1, p}(X)$ in those theorems. The 
reason to use the space $N^{1, p}(\bar{\Omega})$ is that the results become slightly more general at no extra cost.

We also say that $f \in N_{\text {loc }}^{1, p}(\Omega)$ if $f \in N^{1, p}\left(\Omega^{\prime}\right)$ for every open $\Omega^{\prime} \Subset \Omega$.

By a continuous function we always mean a real-valued continuous function, whereas a semicontinuous function is allowed to be extended real-valued, i.e. to take values in the extended real line $\overline{\mathbb{R}}:=[-\infty, \infty]$. We let $f_{+}=\max \{f, 0\}$ and $f_{-}=\max \{-f, 0\}$.

In addition to the assumptions made in the beginning of this section, from now on we assume that $X$ supports a weak $(1, p)$-Poincaré inequality. By Keith-Zhong [17] it follows that $X$ supports a weak $(1, q)$-Poincaré inequality for some $q \in[1, p)$, which was earlier a standard assumption. Throughout the paper we also let $Q \geq 1$ be a real number.

\section{Quasi(super)harmonic functions}

Throughout this section we assume that $\Omega$ is a nonempty open subset of $X$.

We follow Kinnunen-Martio [23], Section 3, making the following definition.

Definition 3.1. A function $u \in N_{\text {loc }}^{1, p}(\Omega)$ is a $Q$-quasiminimizer in $\Omega$ if for all open $\Omega^{\prime} \Subset \Omega$ and all $\varphi \in N_{0}^{1, p}\left(\Omega^{\prime}\right)$ we have

$$
\int_{\Omega^{\prime}} g_{u}^{p} d \mu \leq Q \int_{\Omega^{\prime}} g_{u+\varphi}^{p} d \mu .
$$

A function $u \in N_{\text {loc }}^{1, p}(\Omega)$ is a $Q$-quasisuperminimizer in $\Omega$ if (3.1) holds for all nonnegative $\varphi \in N_{0}^{1, p}\left(\Omega^{\prime}\right)$, and a $Q$-quasisubminimizer in $\Omega$ if (3.1) holds for all nonpositive $\varphi \in N_{0}^{1, p}\left(\Omega^{\prime}\right)$.

A function is a $Q$-quasiminimizer in $\Omega$ if and only if it is both a $Q$-quasisubminimizer and a $Q$-quasisuperminimizer in $\Omega$.

Proposition 3.2. Let $u \in N_{\text {loc }}^{1, p}(\Omega)$. Then the following are equivalent:

(a) The function $u$ is a $Q$-quasisuperminimizer in $\Omega$.

(b) For all open $\Omega^{\prime} \Subset \Omega$ and all nonnegative $\varphi \in N_{0}^{1, p}\left(\Omega^{\prime}\right)$ we have

$$
\int_{\bar{\Omega}^{\prime}} g_{u}^{p} d \mu \leq Q \int_{\bar{\Omega}^{\prime}} g_{u+\varphi}^{p} d \mu
$$


Vol. 81 (2006)

(c) For all nonnegative $\varphi \in N^{1, p}(\Omega)$ with $\operatorname{supp} \varphi \Subset \Omega$ we have

$$
\int_{\varphi \neq 0} g_{u}^{p} d \mu \leq Q \int_{\varphi \neq 0} g_{u+\varphi}^{p} d \mu .
$$

(d) For all nonnegative $\varphi \in \operatorname{Lip}_{c}(\Omega)$ we have

$$
\int_{\varphi \neq 0} g_{u}^{p} d \mu \leq Q \int_{\varphi \neq 0} g_{u+\varphi}^{p} d \mu .
$$

(e) For all nonnegative $\varphi \in \operatorname{Lip}_{c}(\Omega)$ we have

$$
\int_{\operatorname{supp} \varphi} g_{u}^{p} d \mu \leq Q \int_{\operatorname{supp} \varphi} g_{u+\varphi}^{p} d \mu
$$

(f) For all nonnegative $\varphi \in N_{0}^{1, p}(\Omega)$ we have

$$
\int_{\varphi \neq 0} g_{u}^{p} d \mu \leq Q \int_{\varphi \neq 0} g_{u+\varphi}^{p} d \mu .
$$

Remark 3.3. (1) Different authors have used different definitions of quasiminimizers. Kinnunen-Martio [23], Lemmas 3.2, 3.4 and 6.2, gave characterizations of $Q$-quasi(super)minimizers (including (a)-(c)). On the other hand, Ziemer [28], Definition 2.1, defined quasi(super)minimizers (in unweighted $\mathbb{R}^{n}$ ) using condition (f). The characterizations (d) and (e) may be new.

(2) Any of these statements can of course be used to define quasisuperminimizers. The seemingly weakest requirements are made in (e), although (d) may be preferable as a definition. On the other hand, it is sometimes useful to know that (f) holds, in which the strongest requirements are made, this is, e.g., used in the proofs of Lemmas 3.5 and 4.6.

(3) If we omit "super" from (a) and "nonnegative" from (b)-(f) we have a corresponding characterization for $Q$-quasiminimizers. The proof of these equivalences is the same as the proof below.

(4) In (b)-(f) as well as in Definition 3.1 we can replace "nonnegative" with "nonnegative $\mu$-a.e." This follows since any function in $N^{1, p}(\Omega)$ which is nonnegative $\mu$-a.e. is actually nonnegative q.e., and then we can replace such a function by a nonnegative representative in the same equivalence class without changing any of the integrals involved.

(5) Note that some of the integrals occurring in (f) may be infinite. In fact when this happens both sides are always simultaneously $\infty$. 
Proof. (d) $\Rightarrow$ (e) Since $g_{u}=g_{u+\varphi} \mu$-a.e. on $A:=\{x \in \operatorname{supp} \varphi: \varphi(x)=0\}$, we get

$$
\begin{aligned}
\int_{\operatorname{supp} \varphi} g_{u}^{p} d \mu & =\int_{A} g_{u}^{p} d \mu+\int_{\varphi \neq 0} g_{u}^{p} d \mu \\
& \leq \int_{A} g_{u+\varphi}^{p} d \mu+Q \int_{\varphi \neq 0} g_{u+\varphi}^{p} d \mu \leq Q \int_{\operatorname{supp} \varphi} g_{u+\varphi}^{p} d \mu .
\end{aligned}
$$

(e) $\Rightarrow$ (a) Let $\Omega^{\prime} \Subset \Omega$ be open and $\varphi \in N_{0}^{1, p}\left(\Omega^{\prime}\right)$ be nonnegative. Let $\varepsilon>0$. By Theorem 4.8 in Shanmugalingam [27], there is $f \in \operatorname{Lip}_{c}\left(\Omega^{\prime}\right)$ such that $\| f-$ $\varphi \|_{N^{1, p}(X)}<\varepsilon$. We get

$$
\begin{aligned}
\left(\int_{\Omega^{\prime}} g_{u}^{p} d \mu\right)^{1 / p} & \leq Q^{1 / p}\left(\int_{\Omega^{\prime}} g_{u+f}^{p} d \mu\right)^{1 / p} \\
& \leq Q^{1 / p}\left(\int_{\Omega^{\prime}} g_{u+\varphi}^{p} d \mu\right)^{1 / p}+Q^{1 / p}\left(\int_{\Omega^{\prime}} g_{f-\varphi}^{p} d \mu\right)^{1 / p} \\
& \leq Q^{1 / p}\left(\int_{\Omega^{\prime}} g_{u+\varphi}^{p} d \mu\right)^{1 / p}+Q^{1 / p} \varepsilon,
\end{aligned}
$$

where the first inequality is proved as in the proof of $(\mathrm{d}) \Rightarrow$ (e). Letting $\varepsilon \rightarrow 0$ completes the proof of this implication.

(a) $\Rightarrow$ (c) This is Lemma 3.4 in Kinnunen-Martio [23] (after the observation in Remark 3.3 (4) above).

(c) $\Rightarrow$ (f) Let $\varphi \in N_{0}^{1, p}(\Omega)$ and let $\varepsilon>0$. By Lemma 4.10 in Shanmugalingam [27] we can find $f \in N^{1, p}(\Omega)$ with supp $f \Subset \Omega,\|f-\varphi\|_{N^{1, p}(X)}<\varepsilon$ and $\{x \in \Omega: f(x) \neq 0\} \subset\{x \in \Omega: \varphi(x) \neq 0\}$. As in (e) $\Rightarrow$ (a) we get

$$
\left(\int_{\varphi \neq 0} g_{u}^{p} d \mu\right)^{1 / p} \leq Q^{1 / p}\left(\int_{\varphi \neq 0} g_{u+\varphi}^{p} d \mu\right)^{1 / p}+Q^{1 / p} \varepsilon .
$$

Letting $\varepsilon \rightarrow 0$ completes the proof of this implication.

(f) $\Rightarrow$ (d) This is trivial.

(a) $\Rightarrow$ (b) This is similar to the proof of (d) $\Rightarrow$ (e).

(b) $\Rightarrow$ (e) This follows directly after letting $\Omega^{\prime}$ being the open set $\{x: \varphi(x) \neq 0\}$ for $\varphi \in \operatorname{Lip}_{c}(\Omega)$.

By Proposition 3.8 and Corollary 5.5 in Kinnunen-Shanmugalingam [24], a $Q$ quasiminimizer can be modified on a set of capacity zero so that it becomes locally Hölder continuous in $\Omega$. A $Q$-quasiharmonic function is a continuous $Q$-quasiminimizer.

Kinnunen-Martio [23], Theorem 5.3, showed that if $u$ is a $Q$-quasisuperminimizer in $\Omega$, then its lower semicontinuous regularization $u^{*}(x)=\operatorname{ess} \liminf _{y \rightarrow x} u(y)$ is 
also a $Q$-quasisuperminimizer in $\Omega$ in the same equivalence class as $u$ in $N_{\text {loc }}^{1, p}(\Omega)$. Furthermore, $u^{*}$ is $Q$-quasisuperharmonic in $\Omega$. For our purposes we make the following definition.

Definition 3.4. A function $u: \Omega \rightarrow(-\infty, \infty]$ is $Q$-quasisuperharmonic in $\Omega$ if $u$ is not identically $\infty$ in any component of $\Omega, u$ is lower semicontinuously regularized, and $\min \{u, k\}$ is a $Q$-quasisuperminimizer in $\Omega$ for every $k \in \mathbb{R}$.

This definition is equivalent to Definition 7.1 in Kinnunen-Martio [23], see Theorem 7.10 in [23]. (Note that there is a misprint in Definition 7.1 in [23] - the functions $v_{i}$ are assumed to be $Q$-quasisuperminimizers - and that Theorem 7.10 in [23] is incorrectly stated.)

If $u_{j}$ is a $Q_{j}$-quasisuperminimizer in $\Omega, j=1,2$, then, by Corollary 3.8 in [23], $\min \left\{u_{1}, u_{2}\right\}$ is a $\min \left\{Q_{1}+Q_{2}, Q_{1} Q_{2}\right\}$-quasisuperminimizer in $\Omega$; there is also a corresponding result for quasisuperharmonic functions, see Theorem 7.6 in [23]. We will use these facts with $u_{2}$ constant.

By Lemma 5.2 in [23], a quasisuperharmonic function $u$ in $\Omega$ obeys the minimum principle: If $u(x)=\inf _{\Omega} u$ for some $x \in \Omega$, then $u$ is constant in the component of $\Omega$ containing $x$.

We also have a boundary minimum principle of the following type.

Lemma 3.5. Assume that $C_{p}(X \backslash \Omega)>0$ and let $f \in C(\partial \Omega) \cap N^{1, p}(\bar{\Omega})$. Let $u$ be a quasisuperharmonic function in $\Omega$ satisfying $u-f \in N_{0}^{1, p}(\Omega)$. Then $\inf _{\Omega} u \geq$ $\inf _{\partial \Omega} f$.

Note that when $C_{p}(X \backslash \Omega)=0$ the boundary condition $u-f \in N_{0}^{1, p}(\Omega)$ reduces to saying that $u-f \in N^{1, p}(\Omega)$, and in this case the lemma is false.

Proof. Let $m=\inf _{\partial \Omega} f, k=\min \{f, m\}$ and $v=\min \{u, m\}$. Then $v$ is $Q$ quasisuperharmonic in $\Omega$ (for some $Q \geq 1$ ) and $v-k \in N_{0}^{1, p}(\Omega)$, by Lemma 3.6 below. Since $k=m$ on $\partial \Omega, k-m \in N_{0}^{1, p}(\Omega)$, and thus $v-m \in N_{0}^{1, p}(\Omega)$. Hence, using characterization (f) of Proposition 3.2, $\int_{\Omega} g_{v} d \mu \leq Q \int_{\Omega} g_{m} d \mu=0$. Let

$$
w=\left\{\begin{array}{ll}
v-m, & \text { in } \Omega, \\
0, & \text { in } X \backslash \Omega
\end{array} \in N^{1, p}(X) .\right.
$$

It follows that $g_{w}=0$ in $X$, and by the Poincaré inequality $w$ is constant q.e., hence everywhere since $w$ is lower semicontinuously regularized. Thus $v \equiv m$ in $\Omega$.

To make this proof complete we need to prove the following lemma, which we make a little more general for later use in this paper. 
Lemma 3.6. Let $f, h \in N^{1, p}(\Omega)$ and assume that $u-f, v-h \in N_{0}^{1, p}(\Omega)$. Let further $w=\min \{u, v\}$ and $k=\min \{f, h\}$. Then $w-k \in N_{0}^{1, p}(\Omega)$.

If $f, h \in N^{1, p}(\bar{\Omega})$, then this is obvious since we then can assume that $u=f$ and $v=h$ on $\partial \Omega$. However, if $f$ and $h$ only belong to $N^{1, p}(\Omega)$ we need to be a bit more careful. That some care is indeed needed is best illustrated by the fact that if $f$ and $h$ merely belong to $N_{\text {loc }}^{1, p}(\Omega)$, then the lemma is false: Let $f \equiv 0, u(x)=\operatorname{dist}(x, X \backslash \Omega)$, $h \in N_{\text {loc }}^{1, p}(\Omega) \backslash N^{1, p}(\Omega)$ be such that $f \leq h \leq u$ (such an $h$ is easy to find by adding a lot of small oscillations) and $v \equiv h$. Then $w-k=v \notin N^{1, p}(\Omega) \supset N_{0}^{1, p}(\Omega)$.

In fact the lemma is true under the milder assumption $f-h \in N^{1, p}(\Omega)$. To prove this one just use the additional fact that in this case $f, h, k, u, v$ and $w$ all belong to the same coset modulo $N^{1, p}(\Omega)$.

To prove this lemma we need yet another lemma.

Lemma 3.7 (Lemma 5.3 in Björn-Björn [6]). Let $u \in N^{1, p}(\Omega)$ and $v, w \in N_{0}^{1, p}(\Omega)$ be such that $v \leq u \leq w$ q.e. in $\Omega$. Then $u \in N_{0}^{1, p}(\Omega)$.

A standing assumption in [6] was that $\Omega$ is a nonempty bounded open set with $C_{p}(X \backslash \Omega)>0$. However, the proof of this lemma in [6] does not use the boundedness nor the assumption $C_{p}(X \backslash \Omega)>0$ (the lemma is trivial in the case when $\left.C_{p}(X \backslash \Omega)=0\right)$ and thus the lemma holds as stated here.

Proof of Lemma 3.6. That $u, v \in N^{1, p}(\Omega)$ follows directly, and thus $w, k \in N^{1, p}(\Omega)$. Now

$$
w-k=\min \{u-\min \{f, h\}, v-\min \{f, h\}\} \geq \min \{u-f, v-h\} \in N_{0}^{1, p}(\Omega) .
$$

Moreover,

$$
\begin{aligned}
w-k & =\min \{u-f+(f-\min \{f, h\}), v-h+(h-\min \{f, h\})\} \\
& \leq(u-f)_{+}+(v-h)_{+}+\min \{f-\min \{f, h\}, h-\min \{f, h\}\} \\
& =(u-f)_{+}+(v-h)_{+} \in N_{0}^{1, p}(\Omega) .
\end{aligned}
$$

It thus follows from Lemma 3.7, that $w-k \in N_{0}^{1, p}(\Omega)$.

If $Q=1$, "quasi" is omitted from the notation and, e.g., a superminimizer is a 1-quasisuperminimizer.

\section{Weak Kellogg properties}

From now on we assume that $\Omega$ is a nonempty bounded open set with $C_{p}(X \backslash \Omega)>0$.

The main result in this paper is the following theorem. 
Theorem 4.1 (The weak Kellogg property for quasiharmonic functions). Let $f \in$ $C(\partial \Omega) \cap N^{1, p}(\bar{\Omega})$ and let $u$ be a quasiharmonic function in $\Omega$ with $u-f \in N_{0}^{1, p}(\Omega)$. Then

$$
\lim _{\Omega \ni y \rightarrow x} u(y)=f(x) \text { q.e. on } \partial \Omega \text {. }
$$

In the case when $X=\mathbb{R}^{n}$, without much loss, one can just consider quasicontinuous functions $f \in W^{1, p}\left(\mathbb{R}^{n}\right) \cap C(\partial \Omega)$, see the discussion on the space $N^{1, p}(\bar{\Omega})$ in Section 2.

We call this the weak Kellogg property since for a given quasiharmonic function $u$ it says that there is a set $I_{u}$ with $C_{p}\left(I_{u}\right)=0$ such that $u$ has the right boundary values on $\partial \Omega \backslash I_{u}$. The (strong) Kellogg property says that the set $I_{u}$ can be chosen independently of $u$.

Open problem 4.2. Does the (strong) Kellogg property hold for quasiharmonic functions.

Open problem 4.3. Give an example of a boundary point which is regular for $p$ harmonic functions but not for quasiharmonic functions, or prove that no such point exists.

Theorem 4.1 is an immediate consequence of the following result (when applied to both $u$ and $-u$ ).

Theorem 4.4 (The weak Kellogg property for quasisuperharmonic functions). Let $f \in C(\partial \Omega) \cap N^{1, p}(\bar{\Omega})$ and let $u$ be a quasisuperharmonic function in $\Omega$ with $u-f \in$ $N_{0}^{1, p}(\Omega)$. Then

$$
\liminf _{\Omega \ni y \rightarrow x} u(y) \geq f(x) \quad \text { q.e. on } \partial \Omega \text {. }
$$

The following example shows that it is not possible to replace (4.2) by (4.1) in Theorem 4.4, not even for $Q=1$.

Example 4.5. Let $1<p<n, X=\mathbb{R}^{n}$ (with the Lebesgue measure and Euclidean metric) and $\Omega=B(0,1)$. (Note that $\Omega$ is regular for quasiharmonic functions, by, e.g., Remark 2.15 (i) in J. Björn [10], see Definition 5.1.)

Let $\left\{x_{1}, x_{2}, \ldots\right\} \subset \Omega$ be a countable set such that its set of limit points is exactly $\partial \Omega$. We can find $K_{j}$ such that $h_{j}(x)=\max \left\{0,1-K_{j} d\left(x, x_{j}\right)\right\} \in N_{0}^{1, p}(\Omega)$, $\left\|h_{j}\right\|_{N^{1, p}(\Omega)}<2^{-j}$ and $\operatorname{supp} h_{j} \cap \operatorname{supp} h_{k}=\emptyset, k \neq j$. Let $h=\sum_{j=1}^{\infty} h_{j}$. Then $h \in C(\Omega) \cap N_{0}^{1, p}(\Omega)$.

Let $u$ be the continuous solution of the $\mathcal{K}_{h, h}(\Omega)$-obstacle problem, see KinnunenMartio [22], Theorem 5.5. The function $u$ is a continuous superminimizer and thus 
superharmonic in $\Omega$. Let further $f \equiv 0$. Then $u-f \in N_{0}^{1, p}(\Omega)$ but

$$
\limsup _{\Omega \ni y \rightarrow x} u(y) \geq \limsup _{\Omega \ni y \rightarrow x} h(y) \geq 1>0=f(x) \quad \text { for } x \in \partial \Omega .
$$

In order to prove Theorem 4.4 we need the following lemma.

Lemma 4.6. Let $x \in \partial \Omega$ and $B=B(x, r)$. Let $f \in C(\partial \Omega) \cap N^{1, p}(\bar{\Omega})$ be such that $0 \leq f \leq 1$ and $f=1$ on $B \cap \partial \Omega$. Let further $u \leq 1$ be a $Q$-quasisuperminimizer in $\Omega$ with $u-f \in N_{0}^{1, p}(\Omega)$ and let $u \equiv 1$ in $B \backslash \Omega$. Then $u$ is a $Q$-quasisuperminimizer in $B$.

Proof. By Lemma 3.5, $0 \leq u \leq 1$. It is easy to see that $u \in N^{1, p}(B)$. Let $\varphi \in N_{0}^{1, p}(B)$ be nonnegative. We shall prove the inequality

$$
\int_{\varphi \neq 0} g_{u}^{p} d \mu \leq Q \int_{\varphi \neq 0} g_{u+\varphi}^{p} d \mu
$$

Let $\varphi^{\prime}:=\min \{\varphi, 1-u\} \in N_{0}^{1, p}(B)$. Then $\varphi^{\prime}=0$ in $B \backslash \Omega$ and hence $\varphi^{\prime} \in N_{0}^{1, p}(B \cap \Omega)$. As $u$ is a $Q$-quasisuperminimizer in $B \cap \Omega$, we have, using characterization (f) of Proposition 3.2,

$$
\int_{\varphi^{\prime} \neq 0} g_{u}^{p} d \mu \leq Q \int_{\varphi^{\prime} \neq 0} g_{u+\varphi^{\prime}}^{p} d \mu .
$$

As $u+\varphi^{\prime}=\min \{u+\varphi, 1\}$ we have $g_{u+\varphi^{\prime}} \leq g_{u+\varphi}$. Note that $\varphi^{\prime}=0$ if $\varphi=0$. Thus

$$
\begin{aligned}
\int_{\varphi \neq 0} g_{u}^{p} d \mu & =\int_{\varphi^{\prime} \neq 0} g_{u}^{p} d \mu+\int_{\varphi \neq 0=\varphi^{\prime}} g_{u}^{p} d \mu \\
& \leq Q \int_{\varphi^{\prime} \neq 0} g_{u+\varphi^{\prime}}^{p} d \mu+\int_{\varphi \neq 0=\varphi^{\prime}} g_{u+\varphi^{\prime}}^{p} d \mu \leq Q \int_{\varphi \neq 0} g_{u+\varphi}^{p} d \mu .
\end{aligned}
$$

Proof of Theorem 4.4. By Lemma 3.5, $u$ is bounded below in $\Omega$. Let $u_{q}=\min \{u, q\}$ and $f_{q}=\min \{f, q\}, q \in \mathbb{Q}$. Then $u_{q}$ is a quasisuperharmonic function with $u_{q}-f_{q} \in N_{0}^{1, p}(\Omega)$, by Lemma 3.6. Let also $u_{q} \equiv q$ on $X \backslash \Omega$, and $u_{q}^{*}(x)=$ ess $\lim \inf _{y \rightarrow x} u_{q}(y)$ be the lower semicontinuous regularization of $u_{q}$. Since $u_{q}$ already is lower semicontinuously regularized in $\Omega$, we see that $u_{q}=u_{q}^{*}$ in $X \backslash \partial \Omega$.

Let $G_{q}=\{x \in \partial \Omega: f(x)>q\}$, an open set in the relative topology on $\partial \Omega$, and $E_{q}=\left\{x \in G_{q}: \liminf _{\Omega \ni y \rightarrow x} u_{q}(y)<q\right\}$. We want to show that $C_{p}\left(E_{q}\right)=0$. This is immediate if $G_{q}=\emptyset$. Otherwise, since $X$ is separable (which follows from the doubling property), we can write $G_{q}$ as a countable union of balls, $G_{q}=$ $\bigcup_{j=1}^{\infty}\left(B_{q, j} \cap \partial \Omega\right)$. 
By Lemma 4.6, $u_{q}$ is a quasisuperminimizer in $B_{q, j}$, and thus $u_{q}^{*}=u_{q}$ q.e. in $B_{q, j}$. Since $u_{q}^{*}$ is lower semicontinuous we see that

$$
\liminf _{\Omega \ni y \rightarrow x} u_{q}(y)=\liminf _{\Omega \ni y \rightarrow x} u_{q}^{*}(y) \geq u_{q}^{*}(x)=u_{q}(x)=q \quad \text { for q.e. } x \in B_{q, j} \cap \partial \Omega \text {. }
$$

It follows that $C_{p}\left(E_{q}\right)=0$.

Now

$$
\begin{aligned}
I_{u}: & =\left\{x \in \partial \Omega: \liminf _{\Omega \ni y \rightarrow x} u(y)<f(x)\right\} \\
& =\bigcup_{q \in \mathbb{Q}}\left\{x \in \partial \Omega: \liminf _{\Omega \ni y \rightarrow x} u(y)<q<f(x)\right\}=\bigcup_{q \in \mathbb{Q}} E_{q} .
\end{aligned}
$$

Thus $C_{p}\left(I_{u}\right)=0$.

\section{Boundary regularity}

Recall that we assume $\Omega$ to be a nonempty bounded open set with $C_{p}(X \backslash \Omega)>0$.

Definition 5.1. A point $x_{0} \in \partial \Omega$ is regular for quasiharmonic functions if for all $f \in C(\partial \Omega) \cap N^{1, p}(\bar{\Omega})$ and all quasiharmonic functions $u$ in $\Omega$ with $u-f \in N_{0}^{1, p}(\Omega)$,

$$
\lim _{\Omega \ni y \rightarrow x_{0}} u(y)=f\left(x_{0}\right) .
$$

The set $\Omega$ is regular for quasiharmonic functions if all $x \in \partial \Omega$ are regular for quasiharmonic functions.

That a boundary point is regular (i.e. for $p$-harmonic functions) can be defined as in the introduction, or equivalently in a manner similar to Definition 5.1, see BjörnBjörn [6], Theorem 6.1, where several characterizations of regular boundary points can be found. It is immediate that being regular for quasiharmonic functions is a stronger requirement. (We have refrained from using the shorter name quasiregular, which would be tempting but misleading.)

Let us also discuss regularity for quasisuperharmonic functions. Recall that Example 4.5 shows that only one-sided regularity can be obtained in this case.

Definition 5.2. A point $x_{0} \in \partial \Omega$ is regular for quasisuperharmonic functions if for all $f \in C(\partial \Omega) \cap N^{1, p}(\bar{\Omega})$ and all quasisuperharmonic functions $u$ in $\Omega$ with $u-f \in N_{0}^{1, p}(\Omega)$,

$$
\liminf _{\Omega \ni y \rightarrow x_{0}} u(y) \geq f\left(x_{0}\right)
$$


Regularity for superharmonic functions (i.e. 1-quasisuperharmonic functions) is equivalent to regularity for $p$-harmonic functions, see Björn-Björn [6], Theorem 6.1. We do not know if regularity for quasiharmonic functions is equivalent to regularity for quasisuperharmonic functions, but it is clear that the latter implies the former.

Boundary regularity for quasiminimizers has been studied by Ziemer [28] in $\mathbb{R}^{n}$ and J. Björn [10] in metric spaces, they both obtained sufficient conditions for a boundary point to be regular for quasisuperharmonic functions. (J. Björn states her results for quasiminimizers, but the proof of Theorem 2.13 shows that the integral condition therein is sufficient for regularity for quasisuperharmonic functions. The same is true for Theorem 5.3 below.)

J. Björn [11] has recently shown the following result.

Theorem 5.3. Let $G \subset X$ be nonempty and open, and assume that either $X$ is unbounded or $G \neq X$. Then there exist sets $\Omega_{1} \Subset \Omega_{2} \Subset \cdots$, which are regular for quasisuperharmonic functions and are such that $G=\bigcup_{j=1}^{\infty} \Omega_{j}$.

Proposition 5.4. Let $x_{0} \in \partial \Omega$ and $h(x)=-d\left(x, x_{0}\right)$. Then $x_{0}$ is regular for quasisuperharmonic functions if and only if

$$
\liminf _{\Omega \ni y \rightarrow x_{0}} u(y) \geq 0
$$

for all quasisuperharmonic functions $u$ in $\Omega$ with $u-h \in N_{0}^{1, p}(\Omega)$.

It is possible to replace $h$ by any function $k \in C(\partial \Omega) \cap N^{1, p}(\bar{\Omega})$ with $k\left(x_{0}\right)=0$ and $k(x)<0$ for $x \in \partial \Omega \backslash\left\{x_{0}\right\}$. (The proof remains the same.)

Proof. The necessity is trivial. As for the sufficiency, assume that $x_{0}$ is irregular (i.e. not regular) for quasisuperharmonic functions. Then there exist $f \in C(\partial \Omega) \cap$ $N^{1, p}(\bar{\Omega}), \delta \in \mathbb{R}$, and a quasisuperharmonic function $v$ in $\Omega$ with $v-f \in N_{0}^{1, p}(\Omega)$ and

$$
\liminf _{\Omega \ni y \rightarrow x_{0}} v(y)<\delta<f\left(x_{0}\right) .
$$

Assume without loss of generality that $\delta=0$. We can find $M>0$ so that $M h \leq f$ on $\partial \Omega$. Let $w$ be the unique $p$-harmonic function in $\Omega$ with $w-h \in N_{0}^{1, p}(\Omega)$, see Kinnunen-Martio [22], Theorem 3.2, or Shanmugalingam [27], Theorem 5.6. Then the quasisuperharmonic function $u:=\min \{v / M, w\}$ satisfies $u-h \in N_{0}^{1, p}(\Omega)$, by Lemma 3.6. Since

$$
\liminf _{\Omega \ni y \rightarrow x_{0}} u(y) \leq \frac{1}{M} \liminf _{\Omega \ni y \rightarrow x_{0}} v(y)<0
$$

we are done. 
Open problem 5.5. Is it true that if $f \in C(\partial \Omega) \cap N^{1, p}(\bar{\Omega})$ and $v$ is a quasisuperharmonic function in $\Omega$ with $v-f \in N_{0}^{1, p}(\Omega)$, then there is a quasiharmonic function $u \leq v$ in $\Omega$ with $u-f \in N_{0}^{1, p}(\Omega)$ ?

This is true for $Q=1$, i.e. if $v$ is a superharmonic function in $\Omega$ with $v-f \in$ $N_{0}^{1, p}(\Omega)$, then the unique $p$-harmonic function $u$ in $\Omega$ with $u-f \in N_{0}^{1, p}(\Omega)$ satisfies $u \leq v$, see, e.g., Lemma 3.4 in Kinnunen-Martio [22]. The proof there however uses the uniqueness in an essential way.

If the answer to Problem 5.5 were yes, then a straightforward consequence would be the equivalence of the following statements for $x_{0} \in \partial \Omega$ :

(a) $x_{0}$ is regular for quasisuperharmonic functions;

(b) $x_{0}$ is regular for quasiharmonic functions;

(c) for all quasiharmonic functions $u$ in $\Omega$ with $u-h \in N_{0}^{1, p}(\Omega)$, where $h(x)=$ $-d\left(x, x_{0}\right)$, it is true that

$$
\lim _{\Omega \ni y \rightarrow x_{0}} u(y)=0 .
$$

(At present we know that $(\mathrm{a}) \Rightarrow(\mathrm{b}) \Rightarrow(\mathrm{c})$.)

\section{References}

[1] A. Björn, $p$-harmonic measures and the Perron method for $p$-harmonic functions. In Future Trends in Geometric Function Theory RNC Workshop Jyväskylä 2003, Rep. Univ. Jyväskylä Dep. Math. Stat. 92, Univ. Jyväskylä, Jyväskylä 2003, 23-29. Zbl 1046.31002 MR 2058107

[2] A. Björn, Characterizations of $p$-superharmonic functions on metric spaces. Studia Math. 169 (2005), 45-62. Zbl 1079.31006 MR 2139641

[3] A. Björn, Removable singularities for bounded $p$-harmonic and quasi(super)harmonic functions on metric spaces. Ann. Acad. Sci. Fenn. Math. 31 (2006), 71-95. Zbl 05024742 MR 2210110

[4] A. Björn, Regularity classifications of boundary points for $p$-harmonic functions and quasiminimizers. Preprint, Linköping 2006.

[5] A. Björn, and J. Björn, Boundary regularity for $p$-harmonic functions and solutions of the obstacle problem. Preprint, LiTH-MAT-R-2004-09, Linköping 2004.

[6] A. Björn, and J. Björn, Boundary regularity for $p$-harmonic functions and solutions of the obstacle problem. J. Math. Soc. Japan, to appear.

[7] A. Björn, J. Björn and N. Shanmugalingam, The Dirichlet problem for $p$-harmonic functions on metric spaces. J. Reine Angew. Math. 556 (2003), 173-203. Zbl 1018.31004 MR 1971145

[8] A. Björn, J. Björn and N. Shanmugalingam, The Perron method for $p$-harmonic functions. J. Differential Equations 195 (2003), 398-429. Zbl 1039.35033 MR 2016818 
[9] A. Björn, J. Björn and N. Shanmugalingam, Quasicontinuity of Newton-Sobolev functions and density of Lipschitz functions on metric spaces. Preprint, Linköping 2006.

[10] J. Björn, Boundary continuity for quasiminimizers on metric spaces. Illinois J. Math. 46 (2002), 383-403. Zbl 1026.49029 MR 1936925

[11] J. Björn, Approximation by regular sets in metric spaces. Preprint, Linköping 2004.

[12] G. C. Evans, Application of Poincaré's sweeping-out process. Proc. Nat. Acad. Sci. USA 19 (1933), 457-461. Zbl 0006.34902

[13] M. Giaquinta and E. Giusti, On the regularity of the minima of variational integrals. Acta Math. 148 (1982), 31-46. Zbl 0494.49031 MR 0666107

[14] M. Giaquinta and E. Giusti, Quasi-minima Ann. Inst. H. Poincaré Anal. Non Linéaire 1 (1984), 79-107. Zbl 0541.49008 MR 0778969

[15] J. Heinonen, T. Kilpeläinen and O. Martio, Nonlinear Potential Theory of Degenerate Elliptic Equations. Oxford University Press, Oxford 1993. Zbl 0780.31001 MR 1207810

[16] S. Keith, Modulus and the Poincaré inequality on metric measure spaces. Math. Z. 245 (2003), 255-292. Zbl 1037.31009 MR 2013501

[17] S. Keith and X. Zhong, The Poincaré inequality is an open ended condition. Preprint, Jyväskylä 2003.

[18] O. D. Kellogg, Unicité des fonctions harmoniques. C. R. Acad. Sci. Paris 187 (1928), 526-527. JFM 54.0507.02

[19] T. Kilpeläinen, J. Kinnunen and O. Martio, Sobolev spaces with zero boundary values on metric spaces. Potential Anal. 12 (2000), 233-247. Zbl 0962.46021 MR 1752853

[20] J. Kinnunen and O. Martio, The Sobolev capacity on metric spaces. Ann. Acad. Sci. Fenn. Math. 21 (1996), 367-382. Zbl 0859.46023 MR 1404091

[21] J. Kinnunen and O. Martio, Choquet property for the Sobolev capacity in metric spaces. In Proceedings on Analysis and Geometry (Novosibirsk, Akademgorodok, 1999), Sobolev Institute Press, Novosibirsk 2000, 285-290. Zbl 0992.46023 MR 1847522

[22] J. Kinnunen and O. Martio, Nonlinear potential theory on metric spaces. Illinois Math. J. 46 (2002), 857-883. Zbl 1030.46040 MR 1951245

[23] J. Kinnunen and O. Martio, Potential theory of quasiminimizers. Ann. Acad. Sci. Fenn. Math. 28 (2003), 459-490. Zbl 1035.31007 MR 1996447

[24] J. Kinnunen and N. Shanmugalingam, Regularity of quasi-minimizers on metric spaces. Manuscripta Math. 105 (2001), 401-423. Zbl 1006.49027 MR 1856619

[25] P. Koskela and P. MacManus, Quasiconformal mappings and Sobolev spaces. Studia Math. 131 (1998), 1-17. Zbl 0918.30011 MR 1628655

[26] N. Shanmugalingam, Newtonian spaces: An extension of Sobolev spaces to metric measure spaces. Rev. Mat. Iberoamericana 16 (2000), 243-279. Zbl 0974.46038 MR 1809341

[27] N. Shanmugalingam, Harmonic functions on metric spaces. Illinois J. Math. 45 (2001), 1021-1050. Zbl 0989.31003 MR 1879250

[28] W. P. Ziemer, Boundary regularity for quasiminima. Arch. Rational Mech. Anal. 92 (1986), 371-382. Zbl 0611.35030 MR 0823124 
Received October 26, 2004

Anders Björn, Department of Mathematics, Linköpings universitet, 58183 Linköping,

Sweden

E-mail: anbjo@mai.liu.se 